Data Acquisition Kit: DATAQ Instruments' DI-150RS contains all hardware and software needed for two-channel data acquisition and waveform analysis, using the computer's serial port. Users can digitize a transducer's analog output with 12-bit measurement accuracy at rates up to 240 samples per second and record it to a hard disk. Two analog input channels can be software configured as two single-ended channels or one differential channel, both with a gain of 1 or 100. Features include one thermistor input and regulated excitation output. Circle No. 61 on Reader Service Card.

Spectroscopy Catalogs: EG\&G ORTEC ${ }^{3}$ offers two catalogs in one. One catalog on modular pulse-processing electronics and semiconductor radiation detectors covers time and energy spectroscopy, $\mathrm{Ge}$ and $\mathrm{Si}$ detectors, amplifiers, and instruments for time-of-flight, picosecond timing, single-photon counting, and $x$-ray fluorescence. The second catalog, on applied nuclear spectroscopy, offers 32-bit gamma and alpha spectroscopy products, the latest MCAs including the portable DART ${ }^{\mathrm{TM}}$ and digital DSPEC ${ }^{\mathrm{TM}}$, and systems for counting laboratories, water monitoring, and tritium collection.

Circle No. 64 on Reader Service Card.

Thin Film Measurement System: Filmetric's F20 system can measure thickness, refractive index $(n)$, and extinction coefficient $(k)$ of dielectric or semiconductor thin films. Users can measure films from 10 -nm to $50-\mu \mathrm{m}$ thick. Film thickness and wavelength-dependent optical constants are determined by analyzing the sample's reflectivity at 512 wavelengths. Reflectivity data are acquired in less than 1 s; measurement results are available within $2 \mathrm{~s}$. Complex structures are characterized using the MultiLayer reflectivity simulation program integrated in to the F20. Circle No. 65 on Reader Service Card.

Quadrupole ICP-MS: The fully automated ICP-MS PlasmaQuad3 from VG Elemental provides enhanced data quality, detection limits, and accuracy. PlasmaScreen Torch allows operation in a standard mode or a cool plasma mode that eliminates interferences and background noise for elements such as $\mathrm{Na}, \mathrm{K}, \mathrm{Fe}$, or $\mathrm{Ca}$. An ion sampling interface provides sensitivities exceeding $200 \times 10^{6}$ counts/s/ppm, which is one order of magnitude above standard instrument performance. The AutoRange Plus expands the dynamic range to three to eight orders of magnitude.

Circle No. 71 on Reader Service Card.

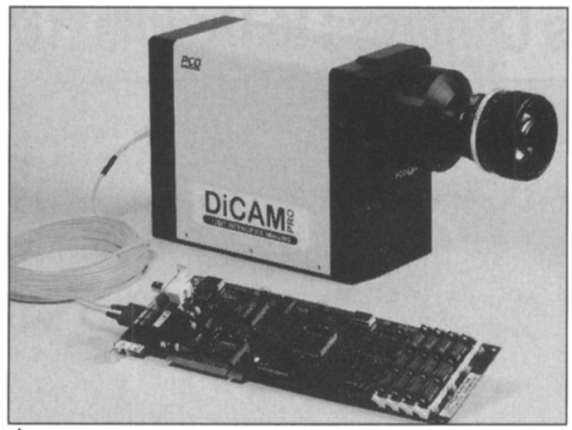

$\Delta$

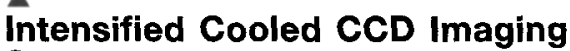

System: The DiCam-Pro from The Cooke Corporation offers a 12-bit dynamic range and a high-resolution $(1,280$ $1,024) \mathrm{CCD}$ sensor. The system provides exposure times down to $3 \mathrm{~ns}$ and features single photon detection and spectral sensitivity from uv to NIR. A serial data stream is transferred via a fiber optic cable connecting the camera and PCIinterface board. The system is suitable for environments exposed to high interference and electrical noise and can be triggered by light or electrical input.

Circle No. 60 on Reader Service Card.

\section{Ultrafiltration and Nanofiltration}

Membranes: Koch Membrane Systems' SelRO membranes can be used in environments not suited for other polymeric membranes. In acid or caustic conditions or solvent environments, the membranes will remain stable and provide a longer useful life compared to conventional ultrafiltration and nanofiltration membranes. Applications include catalyst recovery, precious metals recovery from acids, acid recycling, and COD reduction in chemical wastewater treatment.

Circle No. 68 on Reader Service Card.

Metric Conversions for Pumps and Fluids: Hydraulic Institute's free sixpage booklet contains text and tables of units and procedures to convert pump and fluid data from U.S. customary units to metric units. The booklet includes special conversion factors for space and time, periodic and related phenomena, and heat and electricity. The booklet was derived from ANSI-approved Hydraulic Institute Pump Standards.

Circle No. 66 on Reader Service Card.

Submicron CMOS Readout Multiplexer: Indigo Systems' ISC-9705 was developed for IR focal-plane-array applications. The $320 \times 256$ pixel system features integrated on-chip functionality and is compatible with $p$-on- $n$ detectors fabricated from InSb, HgCdTe, or QWIP technologies indium bump-bonded to the readout device. Snapshot-mode integration affords simultaneous image acquisition of all pixels in the array to eliminate temporal and spatial artifacting during high-speed events.

Circle No. 67 on Reader Service Card.

Four-Module Platform for $300-\mathrm{nm}$ Wafer Processing: The Millennia from GaSonics International uses downstream microwave source technology for $0.18-\mu \mathrm{m}$ photoresist removal, post-etch residue removal, and isotropic etch applications, with extension to $0.12-\mu \mathrm{m}$ devices. The multiple process step capability enables segmentation of long resist removal and clean processes into several short steps. Each process station is optimized for a single process of choice.

Circle No. 69 on Reader Service Card.

Coating Thickness Gauge: The PosiTector $6000^{\circledR}$ FHS from DeFelsko ${ }^{\circledR}$ can be used to measure thick nonmagnetic coatings on ferrous metals. Measuring range is $0-750$ mils, with resolution of $1 \mathrm{mil}$ in the $0-400 \mathrm{mil}$ range and $5 \mathrm{mils}$ in the $400-750 \mathrm{mil}$ range. The FHS probe is one of seven interchangeable probes designed for use with the PosiTector 6000 Series. Functional with the FHS probe are one feature for zeroing on rough substrates and another for measuring within preset thickness limits.

Circle No. 62 on Reader Service Card.

Fine Mesh Ion Exchange Resin Guidelines: Dow Chemical offers a free brochure on using fine mesh ion exchange resins for column separations in chemical and pharmaceutical laboratory applications. DOWEX ${ }^{\mathrm{TM}}$ resins are produced by selectively controlling the suspension polymerization of microporous copolymers of styrene and divinylbenzene technology. The resulting resins consist of whole, spherical beads available with varied crosslinkages and in many particle sizes.

Circle No. 63 on Reader Service Card.

Spectral Mapping Software: EDAX offers Spectral Mapping software for analyzing an entire area of a sample. After collecting spectra, users can create spectra, linescans, and $x$-ray maps from any location in the image. Three setup steps eliminate the need to decide on elements in advance. The software collects $x$-ray spectra at a pre-chosen pixel resolution, saving the information with encoded $x-y$ coordinate information. Separate collections do not have to be performed for images, maps, linescans, and spectra. Circle No. 70 on Reader Service Card. 


\section{Call for the experts in \\ variable temperature SPM}

Talk to us about variable temperature SPM, and you'll be drawing on proven expertise. You'll find we understand your application, whether you're a surface scientist or condensed matter physicist. Our innovative range of SPM products provide high precision and versatility needed for the different applications, and are supported by worldwide distribution and local customer support.

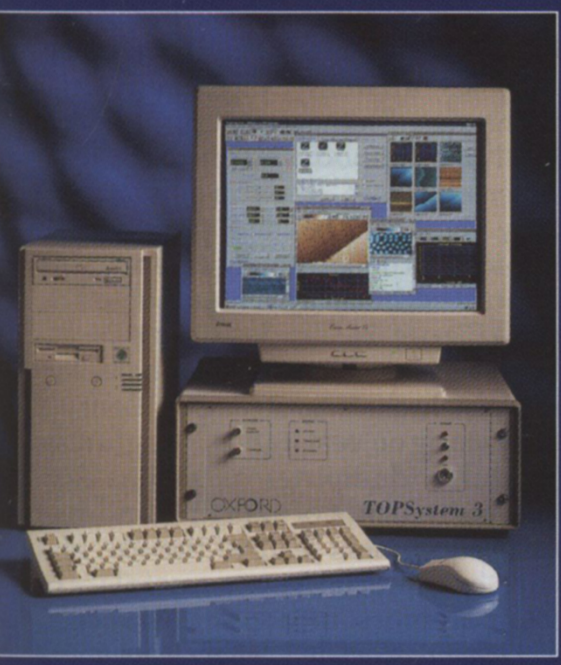

TOPSystem 3 STM Control

\section{System}

- Windows ${ }^{\circledR 5}$ based software for ease of use and flexibility

- multiple parallel processors for high speed

- low noise, high stability tunnelling down to $2 \mathrm{pA}$

- control of Oxford and custom built SPMs.

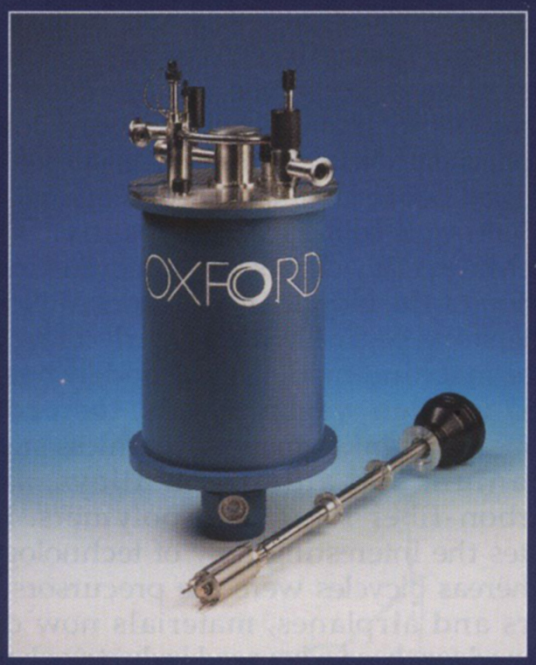

CryosXM

- user configurable for STM, AFM, MFM and NSOM

- operates at variable temperatures below $4 \mathrm{~K}$, and in magnetic fields

- applications include studies of quantum structures and semiconductor devices

- CryosXM/Optistat ${ }^{\mathrm{SXM}}$ package optimised for minimal vibration.

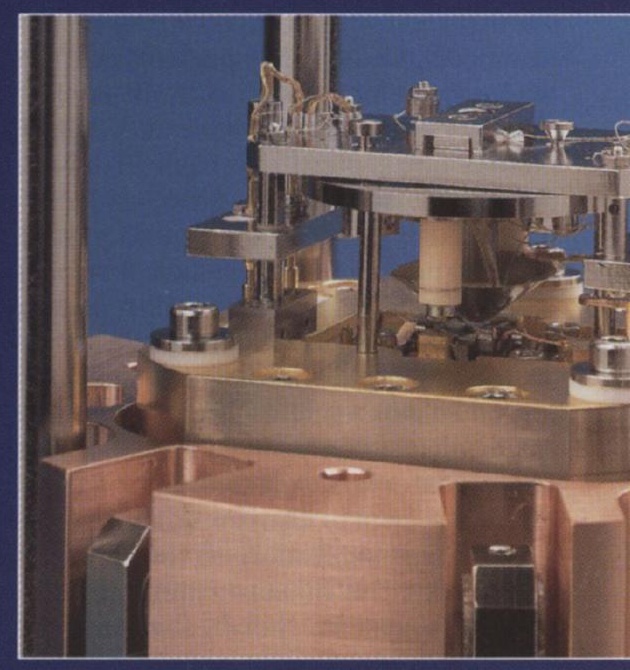

Variable Temperature STM

- surface science applications, including metals from $50-1000 \mathrm{~K}$

- open access allowing deposition during tunnelling

- low mass tip mount allows 'movie rate' in imaging

- same atoms in view during $>200 \mathrm{~K}$ temperature range.

To find out how we can match our expertise to yours, or for more information about any of the featured products, contact us today.

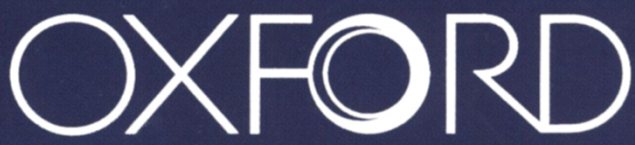

